\title{
Ovarian carcinoma presenting as pneumoperitoneum: Report of a case and review of the literature
}

\author{
ROBERT L. FIORELLI, DO \\ STANLEY D. KOLMAN, DO \\ ANTHONY A. MINISSALE, DO \\ VIVIAN M. BARSKY, Do
}

\begin{abstract}
The exact diagnosis of early stage ovarian carcinoma still remains a very difficult task. Unfortunately, there are no early indicators or serum studies available to screen for this type of malignancy. It usually does not become apparent until metastasis has occurred and the sequelae of its spread have become symptomatic. In the case reported here, the unexpected finding of pneumoperitoneum secondary to metastatic ovarian carcinoma has not been reported in the literature previously.
\end{abstract}

Throughout the years, ovarian carcinoma has been very elusive in its early presentation and diagnosis, because most symptoms of the disease usually are not apparent until metastasis has occurred. While some serum markers, such as $\alpha$-fetoprotein, $\beta$-human chorionic gonadotropin, and carcinoembryonic antigen have been followed in some types of ovarian cancers in postoperative, follow-up studies, no specific serum indicator has been closely linked to the early diagnosis of ovarian carcinoma.

Currently, the usual presenting symptoms of the disease are abdominal pain and distention. Kent and $\mathrm{McKay}^{1}$ found that both of these symptoms were present in more than $50 \%$ of their study population of 349 ovarian cancer patients. The essential point is that the pain usually is secondary to the abdominal distention from ascites. Pain also may be due to distended and ruptured ovary, which results in peritonitis, stretching of the ovarian ligament, or even ovarian torsion. ${ }^{2}$
However, in the case presented here, abdominal pain was present as well as ascites, but the unusual characteristic was pneumoperitoneum secondary to gastric rupture.

\section{Report of case}

A 55-year-old, previously healthy woman presented to the emergency room complaining of abdominal pain and vomiting. She stated that approximately six months earlier, symptoms had started as vague abdominal discomfort associated with some nausea. She recently had noted her abdomen distending. During the past week, this had been accompanied by constipation and vomiting.

Two weeks before her emergency room visit, the patient was examined by her family physician, who noted a pelvic mass. Pelvic untrasonography was ordered and she was scheduled to have a barium enema. However, prior to this being carrried out, the pain had become excruciating, and she came to the emergency room.

The past medical and surgical histories were remarkable only for dilation and curettage for a benign lesion two years earlier. The patient denied any history of smoking or alcohol abuse.

During physical examination, she was in moderate distress from the abdominal pain and was vomiting overtly. The blood pressure was $104 / 70 \mathrm{~mm} \mathrm{Hg}$, the pulse rate was 100 beats per minute, and the respiratory rate was 24 per minute. The tilt test was negative. There was no icterus, but the mucous membranes were extremely dry. There was no evidence of adenopathy, and cardiac, neurologic, and extremity examinations were unremarkable. The lungs had diminished breath sounds in both bases.

There was marked distention of the abdomen, with right upper quadrant and midepigastric guarding and some rigidity. A large, palpable mass extended from the pelvis to the umbilicus. The patient also had markedly 
decreased bowel sounds and a shifting dullness. A large, right-sided adnexal mass extended well out of the pelvis. The uterus was normal to palpation. Digital rectal examination disclosed no masses, but heme-positive stool was present in the ampulla.

Admission CBC findings were as follows: WBC, 9,600/ cu $\mathrm{mm}$; differential count, $52 \%$ polymorphonuclear leukocytes and $34 \%$ band forms, and $14 \%$ lymphocytes; hemoglobin value, $7.1 \mathrm{~g} / \mathrm{dL}$; hematocrit, $22.6 \%$, with decreased red blood cell indices; platelet count, 1,200,000/ cu $\mathrm{mm}$. The serum carcinoembryonic antigen (CEA) level was $5.1 \mathrm{ng} / \mathrm{mL}$; all other laboratory values were within normal limits.

The chest x-ray film (Fig 1) and abdominal survey film revealed an elevated right hemidiaphragm and a pneumoperitoneum. An upper gastrointestinal roentgenographic series using Gastrografin, which was obtained to determine the source of the free intra-abdominal air, revealed an irregular circumferentiated filling defect in the pars media of the stomach, and evidence of mucosal irregularity and luminal narrowing (Fig 2). No free extravasation of the contrast material was noted. At this point, the patient's pelvic ultrasonogram was reviewed; it showed a 9-cm, right-sided ovarian mass and ascites (Fig 3).

After evaluation in the emergency room, the patient was transferred to the intensive care unit, where appro-

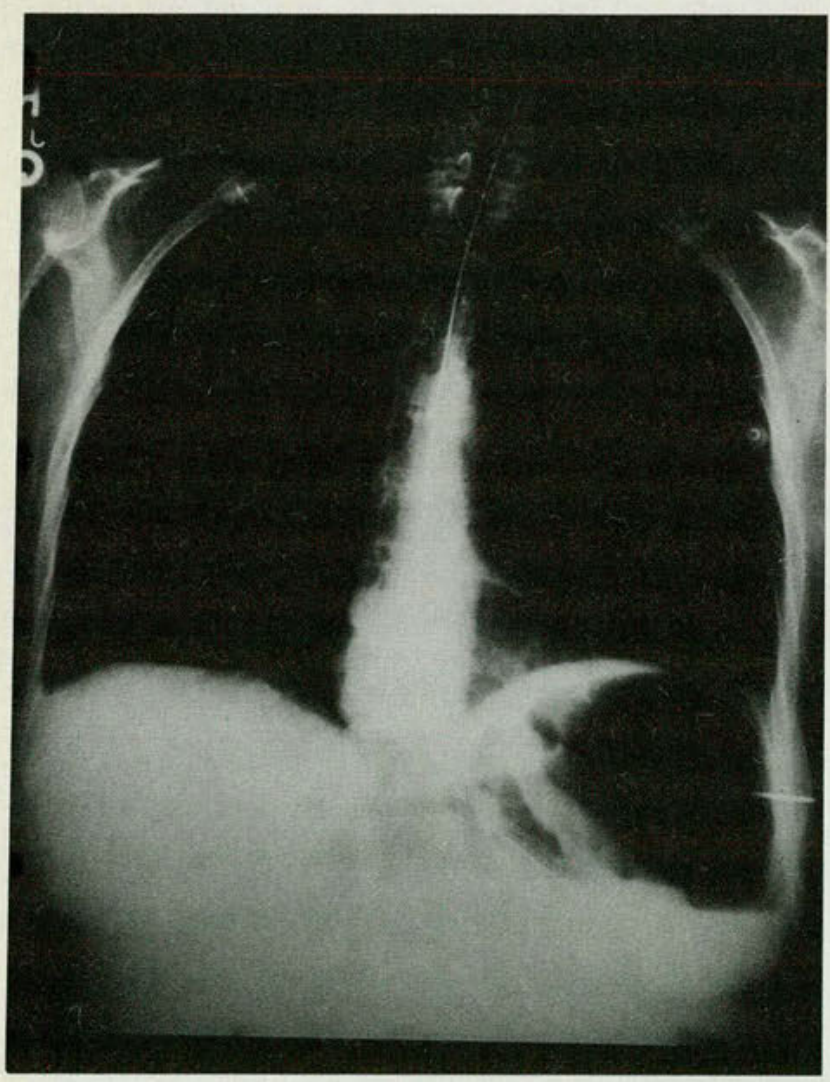

Fig 1. Chest $x$-ray demonstrates elevated right hemidiaphragm and the presence of a pneumoperitoneum. priate stablization therapy was begun. She then was taken to the operating room, where exploratory laparotomy revealed diffuse carcinomatosis, a large tumor mass extrinsically compressing upon the stomach, massive ascites, and a $15-\mathrm{cm}$, solid, right ovarian tumor. The patient also had an area of perforation on the anterior stomach wall in an area of gross tumor invasion. The gastric mucosa was otherwise essentially normal.

Because of her extensive metastases, the patient underwent multiple intra-abdominal biopsy procedures, palliative right salpingo-oophorectomy, and gastrorrhaphy. Pathologic findings were consistent with extensive and partially differentiated adenocarcinoma of ovarian origin. Fig 4, a low-power view of the ovary, demonstrates no normal ovarian tissue and a glandular type of tumor with poor differentiation. Fig 5, a high-power view of the inset in Fig 4, emphasizes the glandular nature of the tumor. Fig 6 demonstrates a normal-appearing gastric mucosa; tumor is present only in the lymphatic channels. In closer view in Fig 7, a solitary tumor cell is apparent in the lymphatic channels. However, on gross examination, tumor was seen to infiltrate the serosa of the stomach and to invade beyond the muscularis, thereby causing perforation.

Postoperatively, the patient's course was complicated by prolonged ileus and septal myocardial infarction. The patient was discharged from the hospital 3 weeks later,

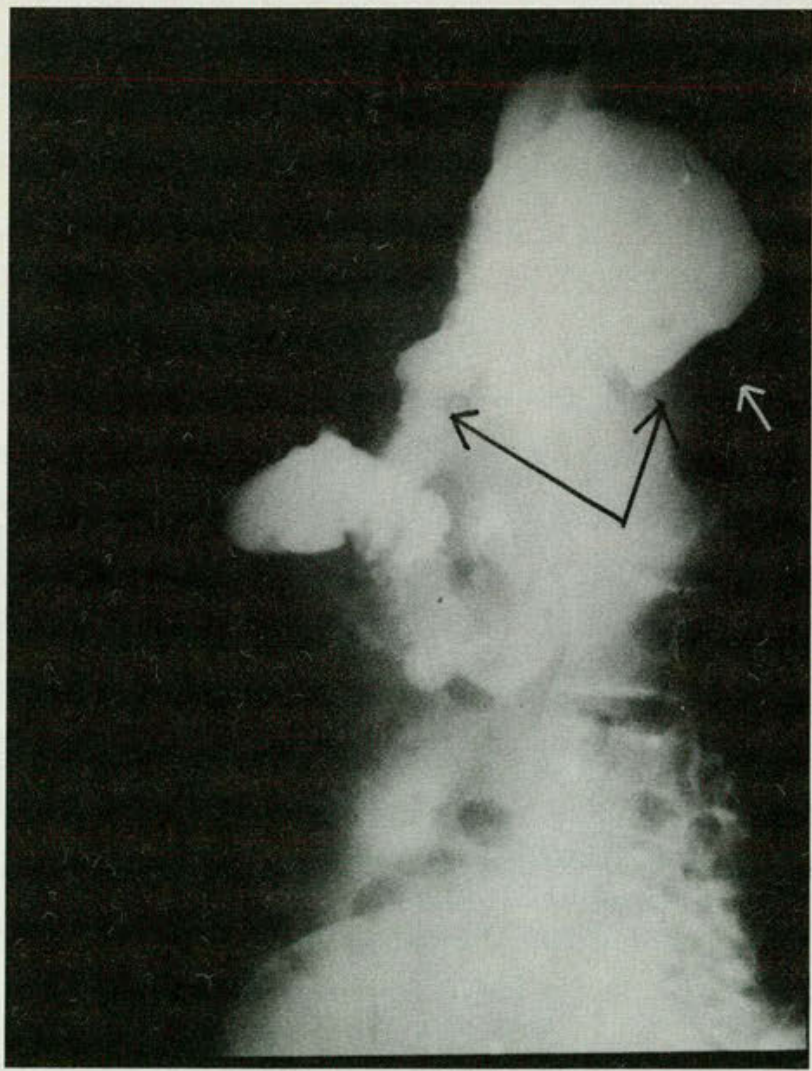

Fig 2. Uppergastrointestinal roentgenogram (with Gastrografin) reveals the intraluminal filling defect in the stomach (arrows). 


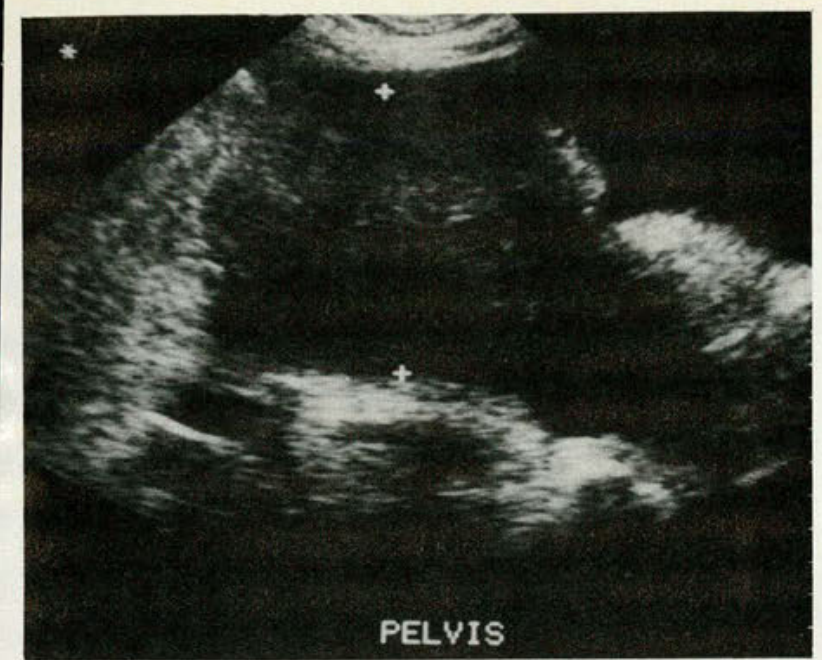

Fig 3. Pelvic ultrasonogram shows a large, right-sided ovarian mass, which is bordered by the crossed bars.

and was to undergo cis-platinum therapy for her metastatic ovarian carcinoma. Unfortunately, after six months of therapy, she died from her illness.

\section{Discussion}

While certain signs and symptoms are common to the disease, the finding of a pneumoperitoneum as a presentation of ovarian carcinoma essentially is unknown. Review of the literature for the past decade has failed to disclose any other reports of pneumoperitoneum as a finding with ovarian cancer. There have been reports only of iatrogenic pneumoperitoneum in the German ${ }^{3}$ and Hungarian ${ }^{4}$ literature; these were induced to demonstrate ovarian metastasis and were not a result of the disease.

Pneumoperitoneum comes from ruptured abdominal viscus and can be secondary to numerous causes. During our patient's workup, the possibility of a Krükenberg tumor of gastric origin with metastasis to the right ovary was considered strongly, because of the apparent intraluminal filling defect in the stomach (Fig 2). This diagnosis obviously was disproved, because the gastric mucosa was essentially normal and the microscopic exam confirmed tumor only in the stomach's lymphatic channels (Figs 6 and 7).

Therefore, in our patient, the severity of carcinomatosis evidently was the cause of her free intraabdominal air. The topical invasion of tumor into the stomach from serosa to mucosa was grossly evident at laparotomy. Also, none of the diagnostic criteria for Krükenberg tumor, as originally described by Novak and Gray ${ }^{5}$ and later alluded to by Metz and associates ${ }^{6}$ and Wong and coauthors ${ }^{7}$ was fulfilled, because no obvious signet ring cells and no sarcomatoid proliferation of the ovarian stroma were noted.

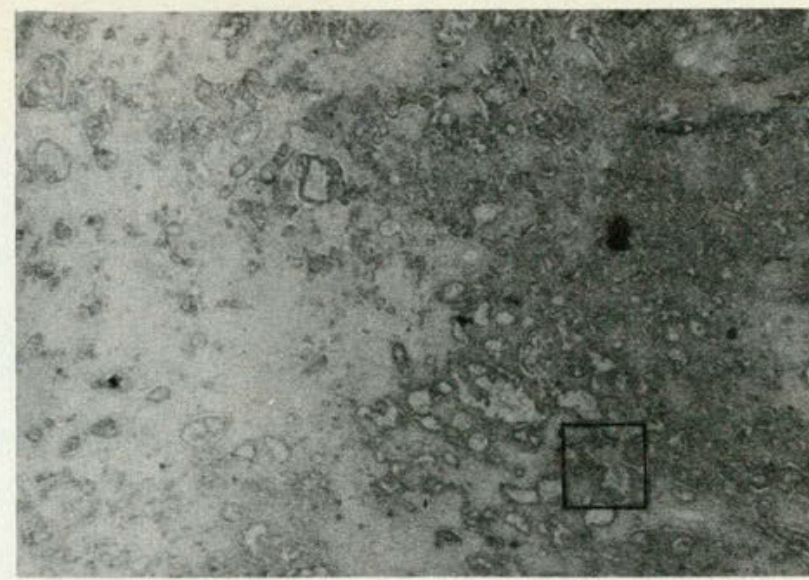

Fig 4. Microscopic view of the ovarian tissue demonstrates a poorly differentiated tumor, with much glandularity and loss of normal tissue architecture (hematoxylin eosin stain magnified $\times 100$ ).

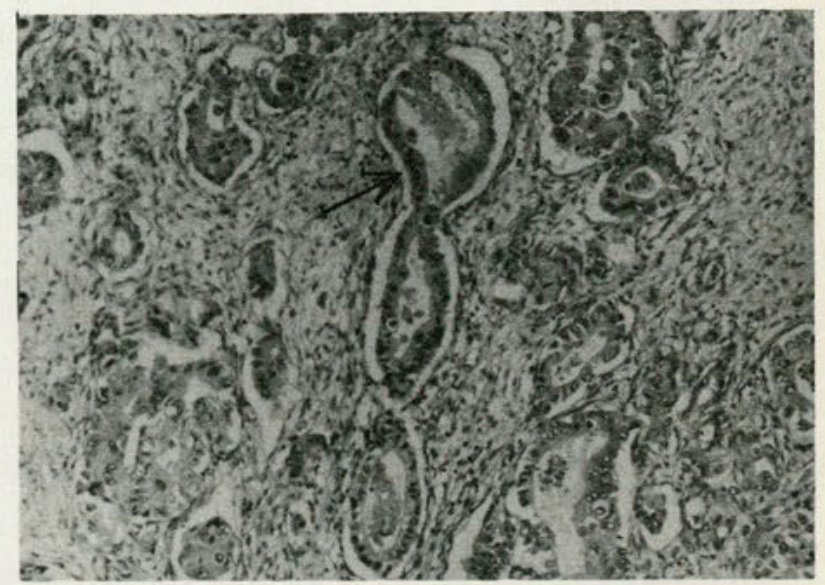

Fig 5. High-power view of the inset in Fig 4 reveals the glandular elements of the tumor (arrow) (hematoxylin eosin stain magnified $\times 400$ ).

Even if the diagnosis of primary Krükenberg tumor is highly suspect, Holtz and $\mathrm{Hart}^{8}$ have demonstrated that the exact pathologic diagnosis of this disease can never be denied unequivocally, because the primary site of the tumor often is unknown, and certain histologic features necessary for the diagnosis vary depending upon the tumor's primary location in the gastrointestinal tract.

Our patient's presenting symptoms were essentially in accord with the standard complaints of abdominal pain and distention, as is the case in $50 \%$ of the patients with ovarian carcinoma. ${ }^{1}$ This statistic is significant because of the extensive metastasis needed for these symptoms to occur.

Ovarian carcinoma remains the leading cause of death among women with gynecologic neoplasms,${ }^{9}$ most likely because of this metastatic nature of the disease at presentation. To date, there ave been no specific diagnostic tests available to detect the tumor in its infant stages. 


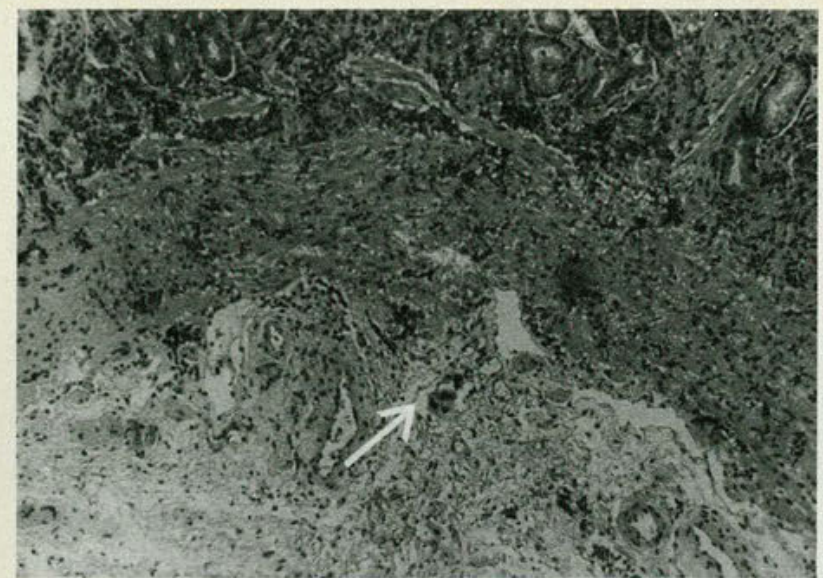

Fig 6. Microscopic overview of the gastric mucosa shows essentially normal tissue of the stomach, except for a solitary tumor cell invading a lymphatic channel (arrow) (hematoxylin eosin stain magnified $\times 100$ ).

Despite numerous investigations for specific serum markers, none has been readily apparent or proved able to identify the disease early in its course. Recently, Bast and coresearchers ${ }^{10}$ have devised a radioimmunoassay using the monoclonal antibody $0 \mathrm{C} 125$, which reacts to a CA125 antigen that is commonly associated with epithelial cell ovarian carcinomas. However, the marker has proved to be a better indicator of response to therapy rather than for use for early diagnosis.

Treatment of the disease also is a very difficult problem. The only apparent fact is that surgery remains the key not only to diagnosis but also to therapy. Roles for radiation and chemotherapy also are advocated, depending on tumor stage and type. Specific guidelines and treatment protocols obviously vary according to each author's personal experience. ${ }^{2,11,12}$

As for our patient, her overall prognosis was quite poor because of her advanced disease. The most ominous sign was the presence of residual tumor mass $>1 \mathrm{~cm}$. In their study population at M.D. Anderson Hospital and Tumor Institute, Smith and Day $^{13}$ found that when the residual tumor mass was $>1 \mathrm{~cm}$ for stage IV disease, the two-year survival rate was only $20 \%$, and the five-year survival rate was $0 \%$.

Therefore, realizing that early diagnosis is very difficult, each of us should maintain a high index of suspicion and do an aggressive diagnostic workup for ovarian carcinoma if the symptom of vague abdominal pain is otherwise unexplainable. ${ }^{11}$

\section{Summary}

Ovarian carcinoma remains a very challenging diagnostic entity. Despite the advent of improved imaging studies, diagnosis is usually not evident un-

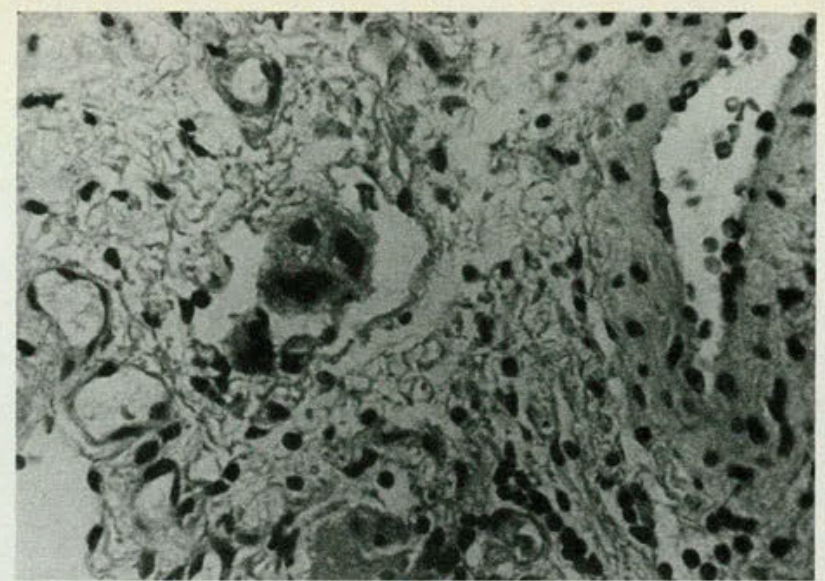

Fig 7. High-power view of the tumor cell located in the stomach's lymphatic channel and bordered by normal gastric tissue (hematoxylin eosin stain magnified $\times 400$ ).

til metastasis has occurred. Because serum markers as screening tests for early stage disease are unavailable, women with abdominal symptoms of unexplained etiology deserve evaluation for ovarian carcinoma.

1. Kent SW, McKay DG: Primary cancer of the ovary. Am J Obstet Gy. necol 1960;80:430-438.

2. Young RC, Knapp RC, Perez CA: Cancer of the ovary, in (ed) DeVita VT, Jr, Hellman S, Rosenberg SA: Principles and Practice of Oncology. JB Lippincott, Philadelphia, 1982, pp 884-908.

3. Perelman VM, Bazina ZA, Titova VA: Tasks in the radiological examination of carcinoma of the uterine body. Radiol Diagn 1979;20:337349.

4. Dofronte Z, Wittman T, Karaczony G: Development of tumor metastasis in the abdominal wall following laparoscopy. Hetil 1977;118:12911292.

5. Novak C, Gray LA: Krükenberg tumor of the ovary: Clinical and pathological study of four cases. Surg Gynecol Obstet 1938;66:157.

6. Metz SA, Karnei RF, Veach SR, et al: Krükenberg carcinoma of the ovary with bone marrow involvement. Report of 2 cases and review of the literature. Obstet Gynecol 1980;55:99-104.

7. Wong PC, Ferenczy A, Fan L-D, et al: Krükenberg tumors of the ovary: Ultrastructural, histochemical, and immunohistochemical studies of 15 cases. Cancer 1986;57:751-760.

8. Holtz F, Hart WR: Krükenberg tumors of the ovary: A clinicopathological analysis of 27 cases. Cancer 1982;50:2438-2447.

9. Smith JP: Ovarian cancer: Update 1984: The current approach to diagnosis and treatment, in The Ovarian Cancer Monograph. Education Division of Febiger McIntyre Inc, Labs, Chicago, 1984.

10. Bast RC Jr, Klug TL, St John E, et al: A radioimmunoassay using a monoclonal antibody to monitor the course of epithelial ovarian carcinoma. N Engl J Med 1983;309:883-887.

11. Rubin P, Bakemeier RF: Cancer of the female genital tract, in Clincal Oncology. New York, American Cancer Society, 1978, pp 114-119. 12. Griffiths CT, Arlan FF: Intensive surgical and chemotherapeutic management of advanced ovarian carcinoma. Surg Clin North Am 1978;58:131-142.

13. Smith JP, Day TG: Review of ovarian carcinoma at the University of Texas System Cancer Center, M.D. Anderson Hospital and Tumor Institute. Am J Obstet Gynecol 1979;135:984-993.

From the Department of Urology at the Osteopathic Medical Center in Philadelphia.

For reprints, address Dr Fiorelli, 9200 Busteton Ave, 202, Philadelphia, PA 19115. 

$\begin{array}{llllllll}P & R & O & F & I & L & E & S\end{array}$
O F

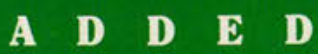
$\begin{array}{llllllll}C & \mathbf{O} & \mathbf{N} & \mathbf{T} & \mathbf{R} & \mathbf{O} & \mathbf{L}\end{array}$

\section{In hypertension...}

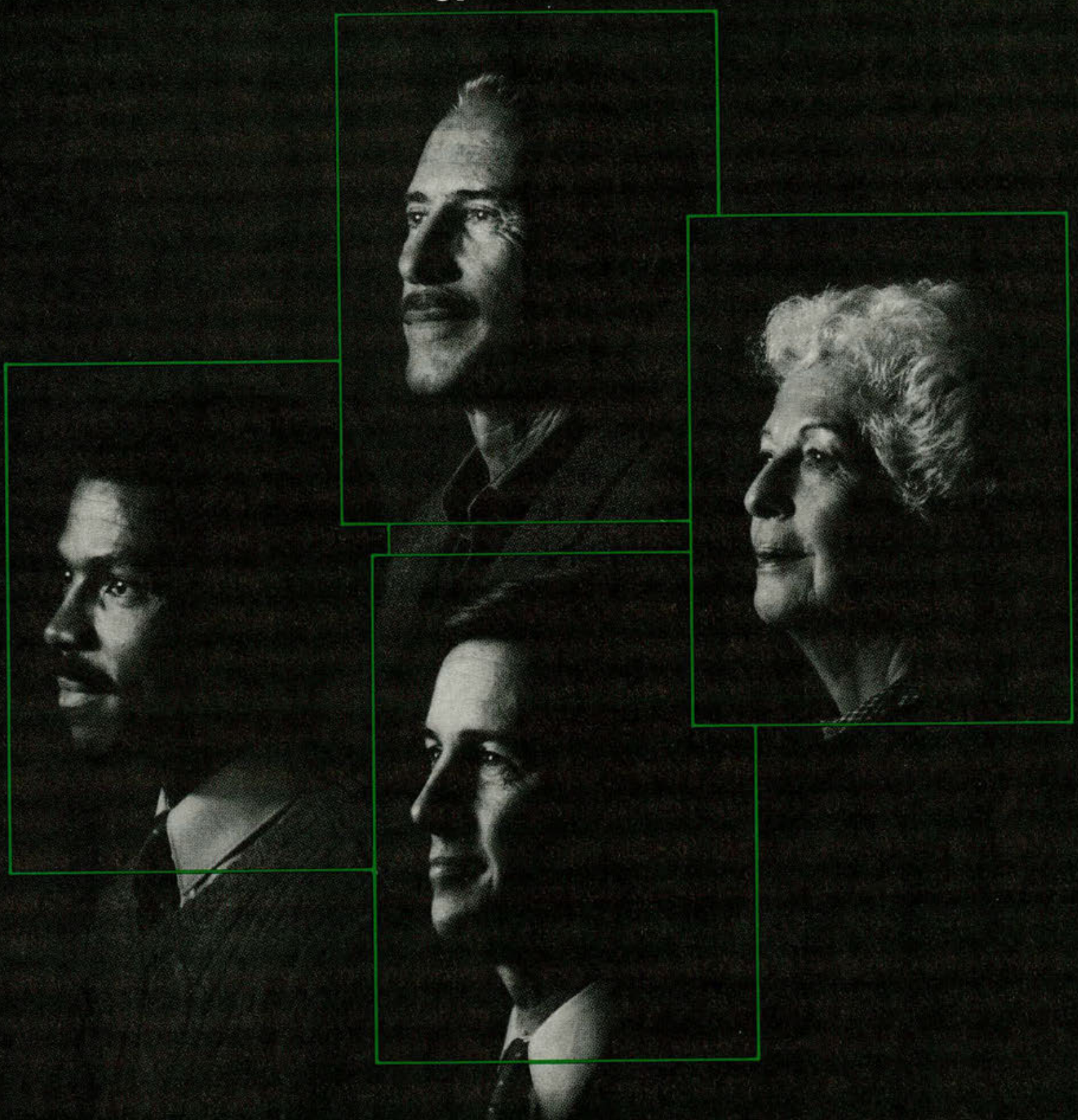

\section{Tenoretic}

Each tablet contains

TENORMIN" (atenoloi) $50 \mathrm{mg}$ or $100 \mathrm{mg}$ and chlorthaldone $25 \mathrm{mg}$

\section{Added control and convenience...} without added side effects in a majority of patients... regardless of age, race, sex, and prior therapy. ${ }^{1}$ 
Added control and convenience without added side effects in a majority of patients...regardless of age, race, sex, and prior therapy. ${ }^{1}$

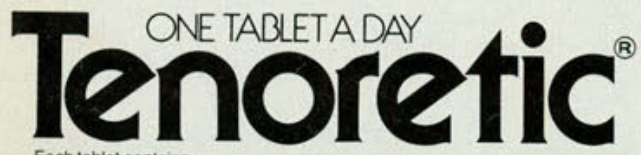

TENORMIN ${ }^{\mathbf{s}}$ (atenolol) $50 \mathrm{mg}$ or $100 \mathrm{mg}$

Please consult complete product information before prescribing. A summary follows:

TENORETIC (atenolol and chlorthalidone) is for the treatment of hypertension. It combines the antihypertensive activity of two agents: a beta 1 -selective (cardioselective) hydrophilic blocking agent Inactive ingredients: magnesium stearate, microcrystalline cellulose, povidone, sodium starch INDICATIONS AND USAGE: TENORETIC is indicated for the treatment of hypertension. This fixed dose combination drug is not indicated for initial therapy of hypertension. If the fixed-dose combination represents the dose appropriate to the individual patient's needs, it may be more CONTRAINDICATIONS: TEate components.

DAIIONS: TENORETIC is contraindicated in patients with: sinus bradycardia, heart hypersensitivity to this product or to sulfonamide-derived drugs.

WARNINGS: Cardiac Failure: Sympathetic stimulation is necessary in supporting circulatory function in congestive heart failure, and beta blockade carries the potential hazard of further depressing myocardial contractility and precipitating more severe failure. In hypertensive patients who have congestive heart failure controlled by digitalis and diuretics, TENORETIC should be

In Patients Without a History of Cardiac Failure:

In Patients Without a History of Cardiac Failure: Continued depression of the myocardium with or symptom of impents over a period of time can, in some cases, lead to cardiac failure. At the first sign be given additional diuretic therapy ure, patients receiving TENORETC should be digitalized and/or adequate digitalization and diuretic therapy. TENORETIC therapy should be withdrawn

Renal and Hepatic Disease and Electrolyte Disturbances: Since atenolol is excreted via the kidneys. TENORETIC should be used with caution in patients with impaired renal function. In patients with renal disease, thiazides may precipitate azotemia. Since cumulative effects may develop in the presence of impaired renal function, if progressive renal impairment becomes evident. TENORETIC should be discontinued.

In patients with impaired hepatic function or progressive liver disease, minor alterations in fluid and electrolyte balance may precipitate hepatic coma. TENORETIC should be used with caution in these (schem

Ischemic Heart Disease: Although not yet reported with atenolol following abrupt cessation of therapy with certain beta-blocking agents in patients with coronary artery disease, exacerbations of angina pectoris and, in some cases, myocardial infarction have been reported. Therefore, such patients should be cautioned against interruption of therapy without the physician's advice. Even in the absence of overt angina pectoris, when discontinuation of TENORETIC is planned, the patient should be carefully observed and should be advised to limit physical activity to a minimum.

Bronchospastic Diseases: PATIENTS WITH BRONCHOSPASTIC DISEASE SHOULD, IN GENERAL, NOT RECEIVE BETA BLOCKERS. Because of its relative beta, -selectivity, however, TENORETIC may respond to, or cannot respond to or cannot tolerate, other antihypertensive treatment. Since betivity is not

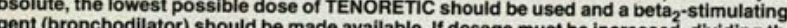
dose should be considered in order to achieve lower peake must be inc

Anesthesia and Major Surgery: As withieve lower peak blood levels.

Anesthesia and Major Surgery: As with all beta-receptor blocking drugs, it may be decided to withdraw TENORETIC before surgery. In this case, 48 hours should be allowed to elapse between last dose and anesthesia. If treatment is continued, care should be taken when using anesthetic agents because of the risk of further depression of the myocardium.

Beta blockers are competitive inhibitors of beta-receptor agonists and their effects on the heart can section on OVERDOSAGE) ypotension) may be corrected with atropine (1-2 mg IV).

Metabolic and Endocrine Effects: TENORETIC may be used with caution in diabetic patients dizziness and sweating may not be significantly affected Atencemia, but other manifestations such as hypoglycemia and, unlike nonselective betantly affected. Atenolol does not potentiate insulin-induce normal levels.

Insulin requirements in diabetic patients may be increased, decreased, or unchanged; latent diabetes mellitus may become manilest during chiorthalidone administration.

Beta-adrenergic blockade may mask certain clinical signs (eg, tachycardia) of hyperthyroidism. Abrupt withdrawal of beta blockade might precipitate a thyroid storm; therefore, patients suspected developing thyrotoxic

Because calcium excretion is decreased by thiazides, TENORETIC should be discontinued before carrying out tests for parathyroid function. Pathologic changes in the parathyroid glands, with hypercalcemia and hypophosphatemia, have been observed in a few patients on prolonged thiazide therapy: however, the common complications of hyperparathyroidism such as renal lithiasis, bone orption, and peptic ulcer

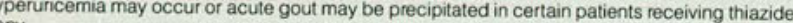

PRECAUTIONS, General-Electrolyte and Fluid Balance Status: Periodic determination of serum . Patients should be observed for clinical signs of fluid or electrolyte imbalance; ie, hyponatrem pypochioremic alkalosis, and hypokalemia. Serum and urine electrolyte determinations are signs or symptoms of fluid and electrolyte imbalance include dryness of the parenteral fluids. Warning letnargy, drowsiness, restlessness, muscle pains or cramps, muscular tatigue, hypotension, oliguria,

tachycardia, and gastrointestinal disturbances such as nausea and vomiting.
Hypokalemia may develop,

concomitant use of corticosteroids orrACTH.

Interference with adequate oral electrolyte intake will also contribute to hypokalemia. Hypokalemia can sensitize or exaggerate the response of the heart to the toxic effects of digitalis (eg, increased
ventricular irritability). Hypokalemia may be avoided or treated by use of potassium supplements or ventricular irritability). Hypokalemia mas
foods with a high potassium content.

Any chloride deficit during thiazide therapy is generally mild and usually does not require specific treatment except under extraordinary circumstances (as in liver disease or renal disease). Dilutional rather than administration of salt except in rare instances when the hyponatremia is life-threatening. In Drug interaction, appropriate replacement is the therapy of choice

Drug Interactions: TENORETIC may potentiate the action of other antihypertensive agents used concomitantly. Patients treated with TENORETIC plus a catecholamine depletor (eg, reserpine) should
be closely observed for evidence of hypotension and/or marked bradycardia which may produce vertigo. syncope, or postural hypotension.

Thiazides may decrease arterial ressponsiveness to norepinephrine. This diminution is not sufficient to preclude the therapeutic effectiveness of norepinephrine. Thiazides may increase the
Lithium generally should not be given with diuretics because they reduce its renal clearance an
add a high risk of lithium toxicity Read circulars for lither add a high risk of lithium toxicity
preparations with TENOREIIC.

Should it be decided to discontinue therapy in patients receiving TENORETIC and clonidine concurrently, the TENORETIC should be discontinued several days before the gradual withdraw

Other Precautions: In patients receiving thiazides, sensitivity reactions may occur with or with history of allergy or bronchial asthma. The possible exacerbation or activation of systernic lupus postsympathectomy patient

Carcinogenesis, Mutagenesis, Impairment of Fertility: Two long-term (maximum dosing dura of 18 or 24 months) rat studies and one long-term (maximum dosing duration of 18 months) mouse
study with atenolol, each employing dose levels as high as $300 \mathrm{mg} / \mathrm{kg} /$ day or 150 times the maxim study with atenolol, each employing dose levels as high as $300 \mathrm{mg} / \mathrm{kg} /$ day or 150

Atenolol was negative in the mouse dominant lethal test, the Chinese hamster in vivo cytogenetic test and the Salmonella typhimurium back mutation test (Ames test). with or without metabolic

Fertility of male or female rats (evaluated at dose levels as high as $200 \mathrm{mg} / \mathrm{kg} /$ day or 100 times th

naximum recommended human dose) was unaffected by atenolol administration.
Use in Pregnancy: Pregnancy Category C. TENORETIC (atenolol and chlorthalidone) was studie for teratogenic potential in the rat and rabbit. Doses of 10,100 and $300 \mathrm{mg} / \mathrm{kg} /$ day were administere
orally to pregnant rats, with no teratologic effects observed. Two studies were conducted in rabbits orally to pregnant rats, with no teratologic effects observed. Two studies were conducted in rabbits
the first study, pregnant rabbits were dosed with 10,100 or $200 \mathrm{mg} / \mathrm{kg} /$ day. No teratologic changes were noted; embryonic resorptions were observed at all dose levels (ranging from approximately 5 5,10 and $25 \mathrm{mg} / \mathrm{kg} /$ day. No teratogenic or embryotoxic effects were demonstrated. It is concluded that the no-effect level for embryonic resorptions is $25 \mathrm{mg} / \mathrm{kg} /$ day (approximately 12.5 times the maximum recommended human dose) or greater. TENORETIC should be used during pregnanc only if the potential benefit justifies the potential risk to the fetus

Atenoloj-Atenolol has been shown to produce a dose-related increase in embryo/fetal resorptio human dose. Although similar effects were not seen in rabbits, the compound was not evaluated in rabbits at doses above $25 \mathrm{mg} / \mathrm{kg}$ or 12.5 times the maximum recommended human dose. There are no adequate and well-controlled studies in pregnant women.

Chlorthalidone - Thiazides cross the placental barrier and appear in cord blood. The use of chlorthalidone and related drugs in pregnant women requires that the anticipated benefits of the dru be weighed against possible hazards to the fetus. These hazards include fetal or neonatal jaundice. Nursing Mothers: It is not established to what extent this drug is excreted in human milk.

most drugs are excreted in human milk, nursing should not be undertaken by mothers receiving

Pediatric Use: Safety and effectiveness in children have not been established.

ADVERSE REACTIONS: TENORETIC is usually well tolerated in properly selected patients. Most adverse effects have been mild and transient. The adverse effects observed for TENORETIC are essentially the same as those seen with the individual components.

Atenolol: The frequency estimates that follow derive from controlled studies in which adverse reactions were either volunteered by the patient (US studies) or elicited. eg, by checklist (foreign studies). The reported frequency of elicited adverse effects was higher for both atenolol and placebc treated patients than when these reactions were volunteered. Where frequency of adverse effects for atenolor and placebo is similar, causal relationship to atenolol is uncertain.

The data present these estimates in terms of percentages: first from the US studies (volunteered side effects) and then from both US and foreign
US STUDIES (\% ATENOLOL-\% PLACEBO):

CARDIOVASCULAR: bradycardia $(3 \%-0 \%)$, cold extremities $(0 \%-0.5 \%)$, postural hypotension

(2\%-1\%), leg pain (0\%-0.5\%)

headedness $(1 \%-0 \%)$, tiredness $(0.6 \%-0.5 \%)$, fatigue $(3 \%-1 \%)$, lethargy $(1 \%-0 \%)$, drowsiness $(0.6 \%-0 \%)$, depression $(0.6 \% \cdot 0.5 \%)$, dreaming $(0 \%-0 \%)$

GASTROINTESTINAL: diarrhea ( $2 \%-0 \%)$, nausea $(4 \%-1 \%)$

RESPIRATORY (See WARNINGS): wheeziness (0\%-0\%), dyspnea $(0.6 \%-1 \%)$
TOTALS US AND FOREIGN STUDIES:

(4\%-5\%), leg pain ( $3 \%-1 \%)$

CENTRAL NERVOUS SYSTEM/NEUROMUSCULAR: dizziness (13\%-6\%), vertigo $(2 \%-0.2 \%)$, ligh headedness $(3 \%-0.7 \%)$, tiredness $(26 \%-13 \%)$, fatigue $(6 \%-5 \%)$, lethargy $(3 \%-0.7 \%)$, drowsiness
$(2 \%-0.5 \%)$, depression $(12 \%-9 \%)$, dreaming $(3 \%-1 \%)$ $2 \%-0.5 \%)$, depression $(12 \%-9 \%)$, dreaming $(3 \%-1 \%)$
GASTROINTESTINAL: diarrhea $(3 \%-2 \%)$. nausea $(3 \%-1 \%)$

RESPIRATORY (see WARNINGS): wheeziness $(3 \%-3 \%)$, dyspnea $(6 \%-4 \%)$

MISCELLANEOUS: There have been reports of skin rashes and/or dry eyes associated with the us have cleared when treatment was withdrawn. Discontinuance of the drug should be considered if an such reaction is not otherwise explicable. Patients should be closely monitored following cessation o therapy

Chlorthalidone: Cardiovascular: orthostatic hypotension; Gastrointestinal: anorexia, gastric irritation vorniting, cramping, constipation, jaundice (intrahepatic cholestatic jaundice). pancreatitis; CNS: aplastic anemia: Hypersensitivity: purpura, photosensitivity, agranulocytosis, thrombocytopenia (vasculitis, cutaneous vasculitis), Lyell's syndrome (toxic epidermal necrolysis); Miscellaneous: hyperglycemia, glycosuria, hyperuricemia, muscle spasm, weakness, restlessness. Clinical trials of
TENORETIC conducted in the United States (89 patients treated with TENORETIC) revealed Potential Adverse Effects.

with adverse effects of atenolol. Nervous System: reversible mental depression progressing to catalonia hallucinations: an acute reversible syndrome characterized by disorientation for time and place. short-term memory loss, emotional lability, slightly clouded sensorium, decreased performance neuropsychometrics; Cardiovascular: intensification of AV block (see CONTRAINDICATIONS); Gastrointestinal; mesenteric arterial thrombosis, ischemic colitis; Hematologic: agranulocytosis, nonthrombocytopenic purpura, thrombocytopenic purpura; Allergic: erythematous rash, fever reversible alopecia, Peyronie's disease.

There have been reports of a syndrome comprising psoriasiform skin rash, conjunctivitis sicca, This syndrome has not been reported with TENORETIC or TENORMIN (atenolol).

Climeal Laboratory Test Findings: Clinically important changes in standard laboratory parameters were not progressive and usually were not associated with clinical manifestations. The DOSAGE AND ADMINISTRATION: Initial dose should be one TENORETIC 50 tablet.on the dosage should be increased to One TENORETIC 100 a day. once a day. Package insert should be consulted for dosage adjustments in cases of 100 tablet impairment of renal function.

(NOW SUPPLIED: TENORETC 50 Tablets (atenolol $50 \mathrm{mg}$ and chlorthalidone $25 \mathrm{mg}$ ). NDC 0310-0115 upplied in bottles of 100 tablets

TENORETIC 100 Tablets (atenolol $100 \mathrm{mg}$ and chlorthalidone $25 \mathrm{mg}$ ), NDC $0310-0117$ (white, round biconvex, uncnated tablets with $\mathrm{ICl}$ on one side and 117 on the other side) are supplied in bottles of
100 tablets.

Protect from heat, light, and moisture. Dispense in well-closed, light-resistant container.

A/12/86

Reference: 1 . TENORETIC Evaluation Program, an open 28-day study of 26,892 hypertensive

patients conducted by more than 7,000 physicians (data on file, ICI Pharma. Wilmington, D
physicians were requested to enter those patients needing more control than provided by 


\section{OHIO UNIVERSITY - COLLEEE OF OSTEOPATHIC MEDICINE \\ Head, Geriatric Medicine/6erontology Section \\ Department of Family Medicine Clinician}

he Ohio University College of Osteopathic Medicine is seeking a physician (0.0. preferred) with an nterest and background in geriatric medicine for a full-time, tenure track clinical faculty position in he Department of Family Medicine as Section Head, Geriatric Medicine/Gerontology.

\section{esponsibilities:}

Provide overall leadership and administrative direction for the Section of Geriatric Medicine/Gerontology, a functional unit within the Department of Family Medicine.

. Participate in the continued development, preparation and delivery of the geriatrics/gerontology curriculum.

3. Provide scheduled clinical services to geriatric patients and promote growth of the Section's clinical programs.

4. Conduct and/or encourage research.

5. Serve on committees and task forces both within and outside the University.

6. Advance the effective use of the interdisciplinary team.

7. Foster the continued development of the Geriatric Clerkship with the College's seven Regional Teaching Centers.

8. Explore sources of grant funding to further program development.

9. Assist with other Section and/or Department functions to be determined in consultation with the Department Chairman.

\section{Qualifications:}

1. Board certified or Board eligible.

2. Ohio licensure (or credentials allowing same).

3. Demonstrated interest in and commitment to Geriatric Medicine.

4. Substantial clinical experience and postgraduate training in geriatrics/gerontology.

5. Ability to work effectively as interdisciplinary team member and to develop the talents of others.

6. Residency and/or fellowship training in geriatrics/gerontology highly desirable.

7. Administrative and teaching experience desirable.

8. Excellent verbal and written communication skills that demonstrate sensitivity, tact, diplomacy, and motivation abilities.

Rank: Assistant/Associate Professor (commensurate with experience/credentials)

Salary: Base Salary - $\$ 28,000-\$ 36,000$ Assistant Professor

$\$ 36,000-\$ 42,000$ Associate Professor

PLUS

Practice Plan Salary - negotiated commensurate with credentials, experience, and service

\section{Research facilities available \\ Rural environment with cultural benefits of university town \\ Excellent fringe benefits}

Closing Date: June 30,1988 , or until filled

Starting Date: September 1, 1988

Application: Send letter of application, Curriculum Vitae, and 3 letters of reference to

Anthony G. Chila, D.0.

Professor and Chairman

Department of Family Medicine

Grosvenor Hall

Ohio University College of Osteopathic Medicine

Athens, Ohio 45701

PH: $614 / 593-2210$

$800 / 345-1440$, ext. 2210 (Ohio)

800/345-1560, ext. 2210 (out-of-state)

Ohio University is an Affirmative Action/Equal Opportunity Employer 
For many osteoarthritic patients whose renal function may be prostaglandin dependent
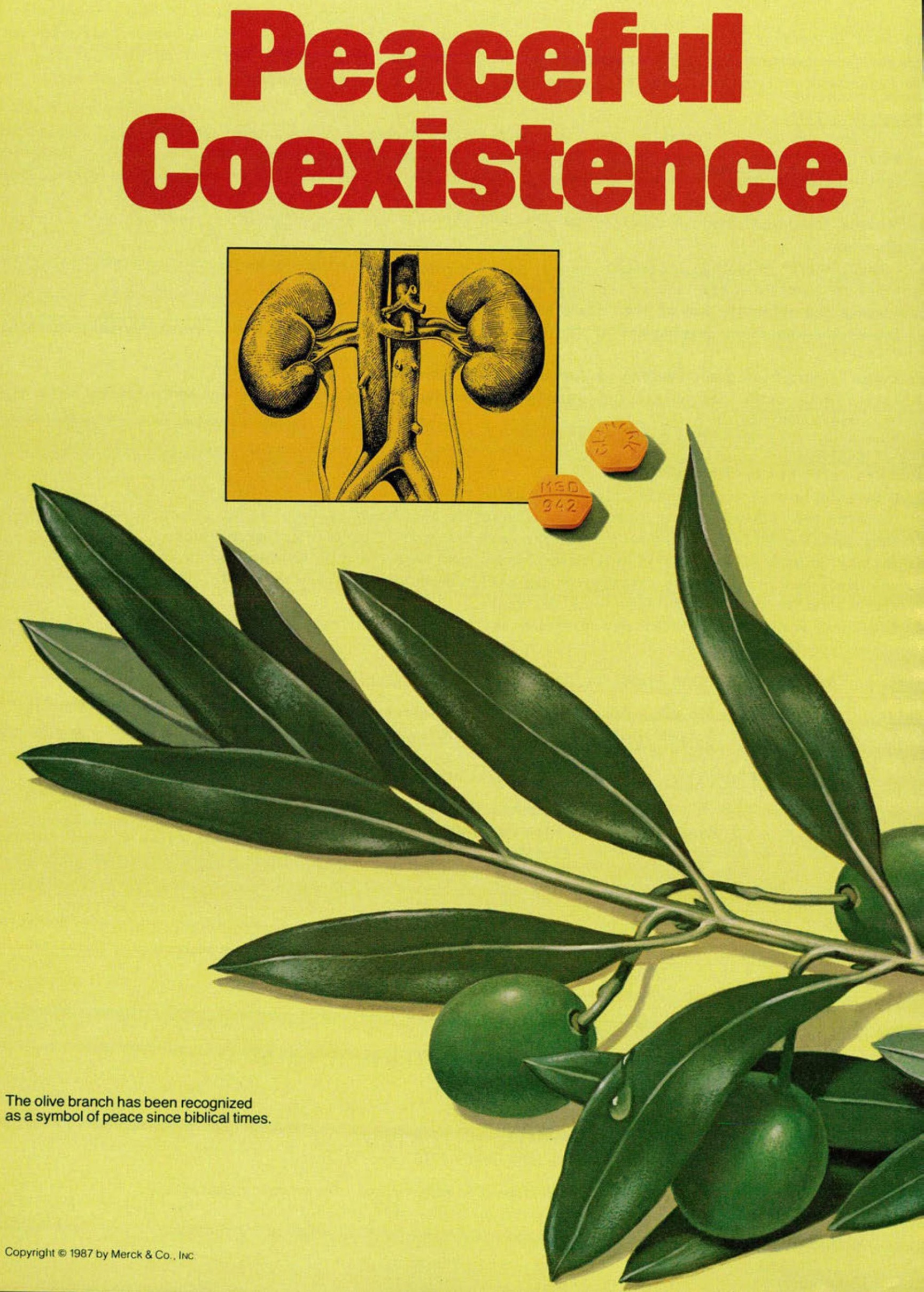


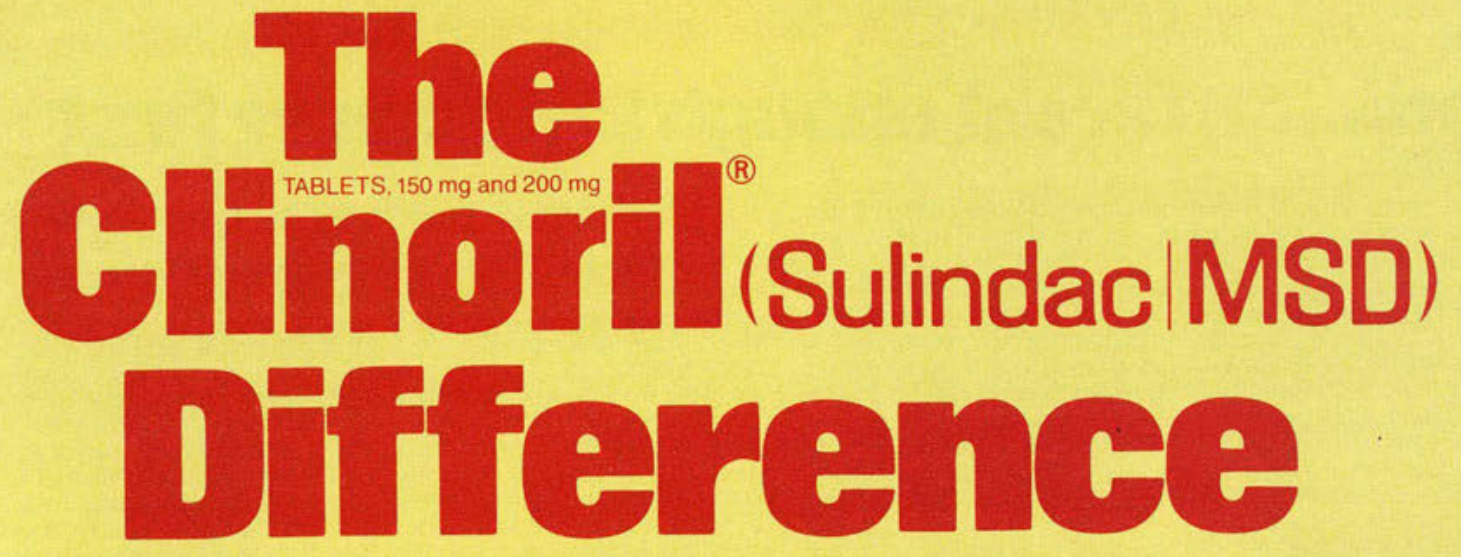

\section{One NSAID that's less likely to compromise osteoarthritic patients whose renal function may be prostaglandin dependent}

In a comparative study, CLINORIL, unlike ibuprofen, did not inhibit renal prostaglandin synthesis or lead to decreases in renal function. ${ }^{1}$

NSAIDs should be administered with caution to patients with renal-prostaglandin-dependent kidney function, such as those with renal or hepatic dysfunction, complications associated with age, extracellular volume depletion, congestive heart failure, sepsis, or concomitant use of any nephrotoxic drug.

CLINORIL is contraindicated in patients who are hypersensitive to this product or in whom acute asthmatic attacks, urticaria, or rhinitis is precipitated by aspirin or other nonsteroida anti-inflammatory agents.

*These observations need further clarification, and, in the interim, sulindac should be used with caution in patients whose renal function may be impaired since renal adverse reactions have been reported with CLINORIL.

1. Ciabattōni, G. et al.: Effects of sulindac and ibuprofen in patients with chronic glomerular disease: Evidence for the dependence of renal function on prostacyclin, N. Engl. J. Med. 310(5): 279-283, February 2, 1984.

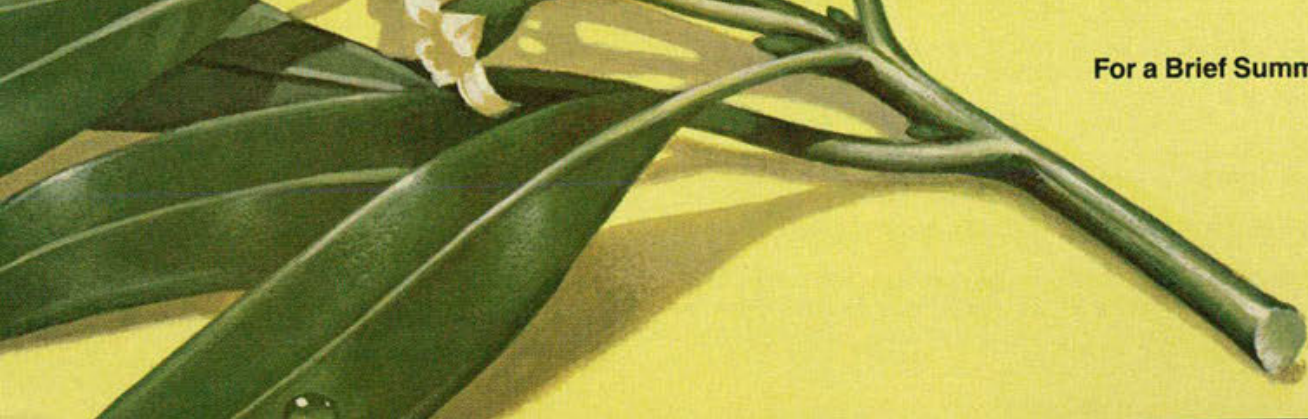




\section{Clinorili..different enough to consider first}

Contraindications: Hypersensitivity to this product; patients in whom acute asthmatic attacks, urticaria, or rhinitis is precipitated by aspirin or other nonsteroidal antiinflammatory agents.

Warnings: Peptic ulceration and gastrointestinal bleeding have been reported. Fatalities have occurred. Gastrointestinal bleeding is associated with higher morbidity and mortality in labile patients, such as the elderly, patients with hemorrhagic disorders, etc. In patients with active gastrointestinal bleeding or an active peptic ulcer, an appropriate ulcer regimen should be instituted, benefits of therapy must be weighed against possible hazards, and the patient's progress carefully monitored; in patients with a history of either upper or lower gastrointestinal tract disease. CLINORIL" (Sulindac, MSD) should be given under close supervision and only after consulting the Adverse Reactions section. Rarely, fever and other evidence of hypersensitivity (see Adverse Reactions), including abnormalities in one or more liver function tests and severe skin reactions, have occurred fatalities have occurred in these patients. Hepatitis, jaundice, or both, with or without fever, may occur usually within the first one to three months of therapy. Determinations of liver function should be considered whenever unexplained fever, rash or other dermato logic reactions, or constitutional symptoms develop; if unexplained fever or other evidence of hypersensitivity occurs, discontinue CLINORIL. Elevated temperature and abnormalities in liver function caused by CLINORIL characteristically have reverted to normal after discontinuation of therapy. CLINORIL should not be reinstituted in such patients.

In addition to hypersensitivity reactions involving the liver, in some patients the findings are consistent with those of cholestatic hepatitis. As with other nonsteroidal anti inflammatory drugs, borderline elevations of one or more liver tests without any other signs and symptoms may occur in up to $15 \%$ of patients. These abnormalities may progress, may remain essentially unchanged, or may be transient with continued therapy The SGPT (ALT) test is probably the most sensitive indicator of liver dysfunction. Meaning ful ( 3 times the upper limit of normal) elevations of SGPT or SGOT (AST) occurred in controlled clinical trials in less than $1 \%$ of patients. A patient with symptoms and/or signs suggesting liver dysfunction, or in whom an abnormal liver test has occurred, should be evaluated for evidence of the development of more severe hepatic reaction while on therapy with CLINORIL. Although such reactions as described above are rare, if abnormal liver tests persist or worsen, if clinical signs and symptoms consistent with liver disease develop, or if systemic manifestations occur (e.g.. eosinophilia, rash, etc.). CLINORIL should be discontinued. In clinical trials, the use of $600 \mathrm{mg} /$ day has been associated with increas cd incidence of mild liver test abnormalities (maximum recommended dosage is $400 \mathrm{mg}$ per day)

Precautions: General-Although the effect on platelet function and bleeding time is less than with aspirin, CLINORIL is an inhibitor of platelet function; therefore, patients who may be adversely affected should be carefully observed when CLINORIL is administered. Pancreatitis has been reported (see Adverse Reactions): should pancreatitis be suspected, the drug should be discontinued and not restarted, supportive medical therapy instituted, and the patient monitored closely with appropriate laboratory studies (e.g. serum and urine amylase, amylase/creatinine clearance ratio, electrolytes, serum calcium, glucose, lipase. etc.): a search for other causes of pancreatitis as well as those conditions which mimic pancreatitis should be conducted. Because of reports of adverse eye findings with nonsteroidal anti-inflammatory agents, it is recommended that patients who develop eye complaints during treatment have ophthalmologic studies. In patients with poor liver function, delayed, elevated and prolonged circulating levels of the sulfide and sulfone metabolites may occur. Such patients should be monitored closely; a reduction of daily dosage may be required.

Edema has been observed in some patients taking CLINORIL. Therefore, as with other nonsteroidal anti-inflammatory drugs. CLINORIL should be used with caution in patients with compromised cardiac function, hypertension, or other conditions predisposing to fluid retention. CLINORIL may allow a reduction in dosage or the elimination of chronic corticosteroid therapy in some patients with rheumatoid arthritis. However, it is generally necessary to reduce corticosteroids gradually over several months in order to avoid an exacerbation of disease or signs and symptoms of adrenal insufficiency. Abrupt withdrawal of chronic corticosteroid treatment is generally not recommended even when patients have had a serious complication of chronic corticosteroid therapy

Renal Effects - As with other nonsteroidal anti-inflammatory drugs, Iong-term administration of sulindac to animals has resulted in renal papillary necrosis and other abnormal renal pathology. In humans, there have been reports of acute interstitial nephritis with hematuria. proteinuria, and occasionally nephrotic syndrome. A second form of renal toxcity has been seen in patients with prerenal and renal conditions leading to a reduction in renal blood flow or blood volume, where renal prostaglandins have a supportive role in the maintenance of renal perfusion. In these patients, the administration of an NSAID may cause a dose-dependent reduction in prostaglandin formation and may precipitate overt renal decompensation. CLINORIL may affect renal function less than other NSAIDs in patients with chronic glomerular renal disease (see CLINICAL PHARMACOLOGY section of Prescribing Information). Until these observations are better understood and clarified, however, and because renal adverse experiences have been reported with CLINORIL (see Adverse Reactions), caution should be exercised when administering the drug to patients with conditions associated with increased risk of the effects of nonsteroidal antiinflammatory drugs on renal function, such as those with renal or hepatic dysfunction. diabetes mellitus, complications associated with advanced age, extracellular volume depletion from any cause, congestive heart failure, sepsis, or concomitant use of any nephrotoxic drug. Discontinuation of NSAID therapy is typically followed by recovery to the pretreatment state. Since sulindac is eliminated primarily by the kidneys, patients with significantly impaired renal function should be closely monitored; a lower daily dosage should be anticipated to avoid excessive drug accumulation.

Use in Pregnancy - Not recommended for use in pregnant women, since safety for use has not been established and because of the known effect of drugs of this class on the human fetal cardiovascular system (closure of the ductus arteriosus) during the third trimester of pregnancy.

Nursing Mothers-Nursing should not be undertaken while a patient is on CLINORIL. It is not known whether sulindac is secreted in human milk: however, it is secreted in the milk of lactating rats.

\section{Use in Children - Safety and effectiveness in children have not been established.} Drug Interactions-DMSO should not be used with sulindac; concomitant administration has been reported to reduce plasma levels of active sulfide metabolite and potentiall reduce efficacy and to cause peripheral neuropathy. Although sulindac and its sulfide metabolite are highly bound to protein, studies with daily doses of $400 \mathrm{mg}$ have shown no clinically significant interaction with oral anticoagulants or oral hypoglycemic agents: however, patients should be monitored carefully until it is certain that no change in their anticoagulant or hypoglycemic dosage is required. Special attention should be paid to patients taking higher doses than those recommended and to patients with renal impair ment or other metabolic defects that might increase sulindac blood levels. Concomitant administration of aspirin significantly depressed the plasma levels of the active sulfide metabolite. Although addition of aspirin did not alter the types of clinical or laboratory adverse experiences, the combination showed an increase in the incidence of gastro intestinal adverse experiences: since addition of aspirin did not have a favorable thera peutic effect, the combination is not recommended. Concomitant administration of diflunisal in normal volunteers resulted in lowering of plasma levels of active sulindac sulfide metabolite by approximately one-third. Probenecid given concomitantly had only a slight effect on plasma sulfide levels, while plasma levels of sulindac and sulfone were increased; sulindac produced a modest reduction in the uricosuric action of probenecid which probably is not significant under most circumstances. Neither propoxyphene hydrochloride nor acetaminophen had any effect on the plasma levels of sulindac or its sulfide metabolite

Adverse Reactions: The following adverse reactions were reported in clinical trials or have been reported since the drug was marketed. The probability exists of a causal rela tionship between CLINORIL" (Sulindac. MSD) and these adverse reactions. The adverse reactions which have been observed in clinical trials encompass observations in 1,865 patients, including 232 observed for at least 48 weeks

Incidence greater than 1\%: Gastrointestinal-Gastrointestinal pain (10\%), dyspepsia * nausea* with or without vomiting. diarrhea,* constipation,* flatulence, anorexia, gastrointestinal cramps. Dermatologic-Rash,* pruritus. Central Nervous SystemDizziness.* headache,* nervousness. SpecialSenses-Tinnitus. Miscellaneous-Edema (see Precautions).

Incidence less than $1 \%$ : Gastrointestinal-Gastritis, gastroenteritis, or colitis: peptic Ilcer: gastrointestinal bleeding: Gl perforation; liver function abnormalities; jaundice, sometimes with fever; cholestasis; hepatitis; pancreatitis (see Precautions); ageusia glossitis. Dermatologic-Stomatitis, sore or dry mucous membranes, alopecia, photosensitivity, erythema multiforme, toxic epidermal necrolysis. Stevens-Johnson syndrome exfoliative dermatitis. Cardiovascular - Congestive heart failure, especially in patients with marginal cardiac function; palpitation; hypertension. Hematologic-Thrombocytopenia; ecchymosis: purpura; leukopenia; agranulocytosis; neutropenia; bone marrow depression, including aplastic anemia; hemolytic anemia; increased prothrombin time in patients on oral anticoagulants (see Precautions). Genitourinary-Urine discoloration: vaginal bleeding; hematuria; proteinuria; crystalluria; renal impairment, including renal failure: interstitial nephritis; nephrotic syndrome. Metabolic-Hyperkalemia. Psychiatric-Depression; psychic disturbances, including acute psychosis. Nervous System - Vertigo, insomnia, somnolence, paresthesia, convulsions, syncope, aseptic meningitis. Special Senses-Blurred vision, visual disturbances, decreased hearing, metallic or bitter taste. Respiratory-Epistaxis. Hypersensitivity Reactions-Anaphylaxis, angioneurotic edema, bronchial spasm, dyspnea, hypersensitivity vasculitis. A potentially fata apparent hypersensitivity syndrome has been reported; this syndrome may include constitutional symptoms (fever, chills), cutaneous findings (rash or other dermatologic reactions - see above), involvement of major organs (changes in liver function, jaundice. pancreatitis, pneumonitis with or without pleural effusion, leukopenia eosinophilia, anemia, renal impairment. including renal failure), and other less specific findings (adenitis. arthralgia, myalgia, fatigue, malaise, hypotension, chest pain, tachycardia).

Causal relationship unknown: Other reactions have been reported in clinical trials or since the drug was marketed but occurred under circumstances where a causal relationship could not be established. However, in these rarely reported events, that possibility cannot be excluded. Therefore, these observations are listed to serve as alerting information to physicians. Cardiovascular-Arrhythmia. Metabolic-Hyperglycemia. Nervous System-Neuritis. Special Senses-Disturbances of the retina and its vasculature. Miscellaneous-Gynecomastia.

Dosage and Administration: CLINORIL should be administered orally twice a day with food. The maximum dosage is $400 \mathrm{mg}$ per day; dosages above $400 \mathrm{mg}$ per day are not recommended. In osteoarthritis, rheumatoid arthritis, and ankylosing spondylitis, the recommended starting dosage is $150 \mathrm{mg}$ twice a day; the dosage may be lowered or raised depending on the response. A prompt response (within one week) can be expected in about one half of patients with osteoarthritis, ankylosing spondylitis, and rheumatoid arthritis: others may require longer to respond. In acute painful shoulder (acute subacromial bursitis/supraspinatus tendinitis) and acute gouty arthritis, the recommended dosage is $200 \mathrm{mg}$ twice a day; after a satisfactory response has been achieved, the dosage may be reduced according to the response. In acute painful shoulder, therapy for 7-14 days is usually adequate. In acute gouty arthritis, therapy for 7 days is usually adequate. Management of Overdosage: Overdosage has been reported, and rarely deaths have occurred. In the event of overdosage, the stomach should be emptied by inducing vomiting or by gastric lavage, and the patient carefully observed and given symptomatic and supportive treatment. Animal studies show that absorption is decreased by the prompt administration of activated charcoal and excretion is enhanced by alkalinization of the urine.

How Supplied: Tablets CLINORIL containing $150 \mathrm{mg}$ or $200 \mathrm{mg}$ sulindac, with cellulose, magnesium stearate, and starch as inactive ingredients, in unit-of-use bottles of 60 and 100 , unit-dose packages of 100 . and bottles of 100 .

\section{unmarked}

